\title{
Study of Intelligent Online Monitoring System for Smart Grid BaseWSNs
}

\author{
Dawei Cheng ${ }^{1}$,Qiang Gao ${ }^{1,2}$,Baohong Geng ${ }^{1}$,YueHan ${ }^{1}$,Guojin Song ${ }^{1}$,Miao Yu ${ }^{1}$,YandanLi ${ }^{1}$ \\ ${ }^{1}$ The Electric Power Research Institute of Liaoning Province Power Company \\ ${ }^{2}$ Shanghai Jiao Tong University (Correspondence should be addressed to: Cheng Da-wei, E-mail: chengdawei@dbdky.com)
}

\begin{abstract}
The substation equipment status monitoring system as an important means to ensure the safe operation of the electrical equipment as well as to enhance the level of production and operation management of substation equipment, and which has become s hot spot for Smart grid researching. In this paper, Through the study of Smart Substation Condition Monitoring System, propose a scheme for Smart substation condition monitoring system based on wireless sensors networks, Discussing the system architecture, solar power system, wireless communication module configuration problem, And the program is applied to $220 \mathrm{kV}$ Smart substation condition monitoring system, through run the system, as well as monitoring data analysis, That the design reduces the overall cost of construction and improve the performance of the system for the substation condition monitoring system, and that has a very important significance for substation equipment condition monitoring system.
\end{abstract}

Keywords-smart grid; online monitoring; wireless sensor networks; link quality evaluation.

\section{INTRODUCTION}

With the rapid development of economy, the grid scale of China's expanding, increasingly high degree of information technology. In this form of development, China State Grid Corp proposed concept of "strong smart grid". In order to ensure network security, and stable operation, be imperative to establish a digital power grid and substation. Intelligent substation technology can solve many difficult problems of conventional substation, one of the more prominent are: interoperability problems, the traditional electromagnetic transformer problems, two circuit, electromagnetic interference caused by control cable, intelligent primary equipment, relay protection maintenance etc.. Widely used in substation automation technology, intelligent, enhanced the ability of the transmission and distribution and grid scheduling, reduces the total cost of the substation construction [1] [2].

Wireless sensor network extends the functionality of existing network, expanding the capacity of human understanding of the world, people use the wireless sensor network direct perception of the objective world, predict the future technology development report, America BusinessWeek and MIT Technology Review respectively listed it as one of the ten technology 21 technology and change the world's most influential twenty-first Century the. The smart grid is the new trend of the development of the electric power industry, it is the integration of information technology, network technology based on traditional grid on (wired and wireless network), control technology, communication technology, power core technology (generation, transmission, and distribution), which transform the original power grid, in order to improve the safety, efficiency and the quality of electric power service system [3] [4]. Wireless network according to network structure can be divided into the center network and no center network. A center of the network needs to default network facilities to operation, installation and maintenance of complex, high cost of operation. Ad hoc wireless sensor network is a network with no center; it does not need the default network facilities can automatic networking operation. Compared with the center of the network, wireless sensor network has the flexibility, fault tolerance and reliability, low cost sensors and rapid development and other characteristics, which can improve the quality of network service. This paper introduces in detail the wireless ad-hoc sensor networks, combining the characteristics of substation automation system communication, the wireless ad-hoc sensor network is applied to substation automation system scheme, and discusses the application problems. [5].

\section{RELATED WORK}

The basic concept of intelligent substation for information collection, transmission, processing, output all the basic characteristics of digital substation, intelligent devices, network communication model and communication protocol, unified automation, operation management etc. The key of intelligent substation construction is to meet the requirements of the communication networks and systems. The IEC61850 standard includes a series of standard general requirements, communication networks and systems in substations function modeling, data modeling, communication protocol, project management and consistency checking etc.. According to IEC61850 standards for the construction of substation communication network and system, comply with the requirements of intelligent substation. [6].smart grid is a core problem is: security, wireless sensor network is a key technology in smart grid, the authors discuss from two aspects to solve the intelligent power grid security scheme. It is a regular place sensors in transmission line sections on the environment difficult, using improved algorithm, transmission line monitoring network health status, risk warning of possible, accurate fault location or locations, in order to achieve the intelligent high efficiency of investigation;[7] secondly, research on smart grid transmission data security, and analyzes the characteristics of data transmission of sensor, combining the digital watermarking technology for sensor data received treatment, 
using multi degeneration to alternating current in the watermark, watermark is embedded in the data before transmission, receiving point received data before the watermark extraction operation, finally verify the data, store and forward legitimate data, discarding the illegal data, so as to guarantee the security of data transmission in smart grid. [8], The smart grid, with two-way, high-speed communication networks and high integration as the foundation, the use of a large number of sensor technology, network technology, efficient control technology, and intelligent decision support system to transform the traditional power grid, which has better reliability, environmental protection, user interaction, economic benefit, efficiency and environment friendly, intelligent target.

\section{EXPERIMENT}

Hardware platform of experimental is the MicaZ node developed by Crossbow Company; its working band is the 2.4-2.4835 GHz ISM band. The module has DSSS radio which has the 250kbps maximum data transfer rate; its communications module is high-performance wireless chip CC2420 [9].

The software platform is TinyOS [10]. TinyOS is an open-source, open-community effort. As such, the design direction of TinyOS is directed as much as possible by working groups comprised of people with diverse experiences and backgrounds but a common interest in TinyOS.

\section{A. Communication measurement}

A remarkable feature of the wireless sensor network is a network formation does not depend on the preset basic facilities, nodes at any time, any place automatically connected to form a network, so as to realize the interactive information between nodes and forwarding. Specifically, network routing, network take temporary topology does not require external interference, but by then the situation to take self-organization, and self-adjusted according to changes in the environment.

This paper, the experiments were carried out in outdoor, communications links were measured using different distances between nodes. Experiment uses 20 WSNs nodes, divided ten groups. In the same distance, the relationship between $\mathrm{d}$ and prrfor forward link and reverse link.

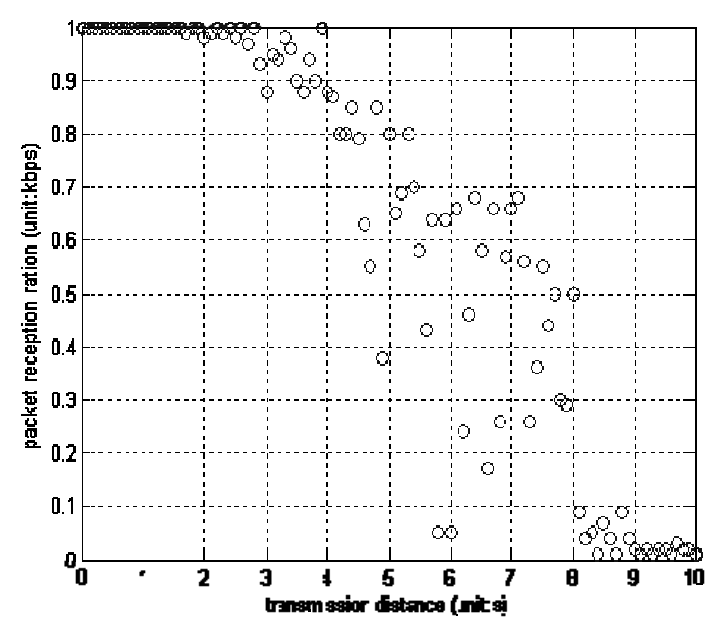

Figure 1.d and prrrelationship

The smart grid is not only is an infrastructure system, but also complete information architecture. Through the communication technology, the sensor technology can be real-time monitoring of power grid operators, power customer, power enterprise assets, according to the collected information can take "on demand" measures to improve the Power Grid Corp's business efficiency, management level and service quality. Compared with the traditional advantages of smart grid monitoring system is very obvious, the level of detail surveillance and monitoring it's have the qualitative change, for the grid system painted in a more comprehensive and detailed operation view, on the analysis of related parameters change can take measures to optimize the power business and. Smart grid has changed the traditional mode of power management is adopted that limited, based on the time lag of the information from the root, it largely help electric power enterprises to achieve more precise operation scheme and intelligent management strategy.

This paper has made the following statement: Theodore $\mathrm{S}$ analysis the underlying link for wireless sensor network communication model of lognormal shadow:

$$
P L(d)=P L\left(d_{0}\right)+10 \beta \log \left(\frac{d}{d_{0}}\right)+X_{\sigma}
$$

From (1) can be received by the receiver of the power:

$$
P_{\text {recv }}=P_{\text {trans }}-P L(d)
$$

From(2) can be calculated signal to noise ratio in the receiving node (SNR) as:

$$
S N R_{(d b m)}=P_{r e c v(d b m)}-N_{(d b m)}
$$

From(3) can be calculatedthe bit error rate formula is:

$$
P_{e}=\frac{1}{2} \exp ^{-\frac{1}{2} S N R}
$$

From(4) can be calculated the SNR (signal-to-ratio) function; the expression is: 


$$
f(\lambda)=\left(1-\frac{1}{2} \exp ^{-\frac{\lambda B_{N}}{2 R}}\right)^{8 \rho F}
$$

Through the above analysis, from the recursive computation, just to find the nodes meet the following conditions: in the end to end link, if a power series for each additional, to deliver the fastest rate of increase.

\section{System TESTAND PERFoRmanceEvaluation}

Wireless sensor networks are application specific, in different application environment, and the theoretical results and corresponding to be adjusted or improved, can satisfy the requirements of the site monitoring. The smart grid is a technical innovation to the traditional network, smart grid has more requirements on safety, such as power generation, power transmission and substation safety etc.. This chapter combines the localization technology to solve the transmission line of smart grid security. Due to the particularity of the transmission line of smart grid, traditional localization algorithms and cannot directly use, so need to suit one's measures to local conditions to be improved.

AODV is a kind of source driven routing protocol. When a node needs to other nodes in the network transmission of information, if not to reach the goal of routing nodes, you must first to form a RREQ (multicast routing request) message. The network layer address in the RREQ message recording start node and the target node, the adjacent node receives the RREQ, first determine the target node is yourself. If it is, then to the initiating node sends RREP (route reply); if not, it is first in the routing table lookup is to reach the goal of routing nodes, if any, to the source node unicast RREP, otherwise continue forwarding RREQ search.

Loss calculation formula is as follows:

$$
\operatorname{Loss}(j)=\frac{\text { NumberDrops }(j)}{\text { NumberTimes }(j)}
$$

From the simulation experiment, The conclusion can be drawn: REEOA-AODV considers the lower-link communication characteristics of the non-symmetry, so its throughput is even higher than the ETX-AODV.

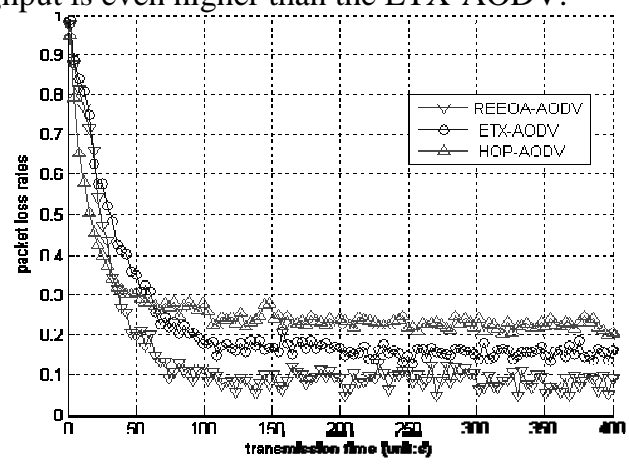

Figure 2.Loss rates vs $\mathrm{T}$

\section{V.CONCLUSIONS}

Wireless sensor network extends the functionality of existing network, expanding the capacity of human understanding of the world, people use the wireless sensor network direct perception of the objective world, predict the future technology development report, America BusinessWeek and MIT Technology Review respectively listed it as one of the ten technology 21 technology and change the world's most influential twenty-first Century the. The smart grid is the new trend of the development of the electric power industry, it is the integration of information technology, network technology based on traditional grid on (wired and wireless network), control technology, communication technology, power core technology (generation, transmission, and distribution), which transform the original power grid, in order to improve the safety, efficiency and the quality of electric power service system.

\section{REFERENCES}

[1] Singhal, A., Software models for Smart Grid [J],Software Engineering for the Smart Grid (SE4SG), 2012 International Workshop on,pp.3-3 June 2012.

[2] Datta, A.,Accelerated deployment of Smart Grid technologies in India - Present scenario, challenges and way forward [C], IEEE Transactions on Industrial Informatics,6(4), pp.19-22 Feb. 2014.

[3] Krco, S. ; Tsiatsis, V. ; Matusikova, K. ; Johansson, M. ; Cubic, I. ; Glitho, R.Mobile Network Supported Wireless Sensor Network Services [J], IEEE Wireless Communications,17(5), pp. 44 49,2010.

[4] Weiwei Fang,Feng Liu,Fangnan Yang, Lei Shu,Nishio, S., Energy-efficient cooperative communication for data transmission in wireless sensor networks[C], Mobile Adhoc and Sensor Systems, 2007. MASS 2007. IEEE International Conference on, 2007 , Page(s): 1- 3

[5] Akshay, N. ; Kumar, M.P. ; Harish, B. ; Dhanorkar, S.,An efficient approach for sensor deployments in wireless sensor network [C], Emerging Trends in Robotics and Communication Technologies (INTERACT), 2010 International Conference on,pp.350 - 355

[6] Ichihara, Y., Challenges for engineers in the electric power industry[J], Power Engineering Review,20(12), pp. 3-6,2000.

[7] Galli, S.,Scaglione, A.,Zhifang Wang, For the Grid and Through the Grid: The Role of Power Line Communications in the Smart Grid [J].Proceedings of the IEEE,99(6), pp. 998 - 1027,2011.

[8] Processing in Sensor Networks, 2009. IPSN 2009[C], pp. 429 - 430, 2009.

[9] D. Ebehard and E. Voges, "Digital single sideband detection for interferometric sensors," presented at the 2nd Int. Conf. Optical Fiber Sensors, Stuttgart, Germany, 1984.

[10] Hyunwoo Joe, Demo abstract: A high-fidelity sensor network simulator using accurate CC2420 model, Information Processing in Sensor Networks, 2009. IPSN 2009. International Conference on [C], pp. 429 - 430, 2009.

[11] Handong Ye, "TiNy threads on BlueGene/P: Exploring many-core parallelisms beyond The traditional OS "Workshops and Phd Forum (IPDPSW), 2010 IEEE International Symposium on.19-23 April 2010 